\title{
Spectra of random regular hypergraphs
}

\author{
Ioana Dumitriu \\ Department of Mathematics, \\ University of California San Diego \\ La Jolla, CA 92093, U.S.A. \\ idumitriu@ucsd.edu
}

\author{
Yizhe Zhu \\ Department of Mathematics, \\ University of California San Diego \\ La Jolla, CA 92093, U.S.A. \\ yiz0840ucsd.edu
}

Submitted: May 18, 2019; Accepted: Jul 13, 2021; Published: Jul 30, 2021

(C) The authors. Released under the CC BY-ND license (International 4.0).

\begin{abstract}
In this paper, we study the spectra of regular hypergraphs following the definitions from Feng and Li (1996). Our main result is an analog of Alon's conjecture for the spectral gap of the random regular hypergraphs. We then relate the second eigenvalues to both its expansion property and the mixing rate of the nonbacktracking random walk on regular hypergraphs. We also prove the spectral gap for the non-backtracking operator of a random regular hypergraph introduced in Angelini et al. (2015). Finally, we obtain the convergence of the empirical spectral distribution (ESD) for random regular hypergraphs in different regimes. Under certain conditions, we can show a local law for the ESD.
\end{abstract}

Mathematics Subject Classifications: 60C05, 60B20

\section{Introduction}

Since their introduction in the early 1970s (see, for example, Berge's book [5]), hypergraphs have steadily risen to prominence, both from a theoretical perspective and through their potential for applications. Of the most recent fields to recognize their importance we mention machine learning, where they have been used to model data [45], including recommender systems [39], pattern recognition [27] and bioinformatics [40].

As with graphs, one main feature for the study is graph expansion; e.g., studies of regular graphs $[1,32,17,2,6]$, where all vertices have the same degree $d$, and quasiregular graphs (e.g., bipartite biregular [8, 9], where the graphs are bipartite and the two classes are regular with degrees $d_{1}$, respectively, $d_{2}$; or preference models and $k$ frames [42], which generalizes these notions). The key property for graph expansion is fast random walk mixing. There are three main perspectives on examining this property: vertex, edge, and spectral expansion [10]; the latter of these, the spectral gap, is the 
most desirable feature as it controls the others (the bounds on vertex and edge expansion generally involve the second eigenvalue of the Laplacian of the graph).

For general, connected, simple graphs (possibly with loops), the Laplacian is a scaled and shifted version of the adjacency matrix $A=\left(A_{i j}\right)_{1 \leqslant i, j \leqslant n}$, where $A_{i j}=1$ if and only if $i$ and $j$ are connected by an edge and 0 otherwise. The Laplacian is defined by $L=I-D^{-1 / 2} A D^{-1 / 2}$, where $D$ is the diagonal matrix of vertex degrees.

As mentioned before, spectral expansion of a graph involves the spectral gap of its Laplacian matrix; however, in the case of regular or bipartite biregular graphs, looking at the adjacency matrix or the Laplacian is equivalent (in the case of the regular ones, $D$ is a multiple of the identity, and in the case of the bipartite biregular ones, the block structure of the matrix ensures that $D^{-1 / 2} A D^{-1 / 2}=\frac{1}{\sqrt{d_{1} d_{2}}} A$ ). For regular and bipartite biregular graphs, the largest (Perron-Frobenius) eigenvalue of the adjacency matrix is fixed (it is $d$ for $d$-regular graphs and $\sqrt{d_{1} d_{2}}$ for bipartite biregular ones). So for these special cases, the study of the second largest eigenvalue of the adjacency matrix is sufficient. As we show here, this will also be the case for $(d, k)$-regular hypergraphs.

The study of the spectral gap in $d$-regular graphs with fixed $d$ had a first breakthrough in the Alon-Boppana bound [1], which states that the second largest eigenvalue $\lambda:=$ $\max \left\{\lambda_{2},\left|\lambda_{n}\right|\right\}$ satisfies $\lambda \geqslant 2 \sqrt{d-1}-o(1)$. Later, Friedman [17] proved Alon's conjecture [1] that a uniformly chosen random $d$-regular graphs have $\lambda \leqslant 2 \sqrt{d-1}+\epsilon$ for any $\epsilon>0$ with high probability, as the number of vertices goes to infinity. Recently, Bordenave [6] gave a new proof that $\lambda_{2} \leqslant 2 \sqrt{d-1}+\epsilon_{n}$ for a sequence $\epsilon_{n} \rightarrow 0$ as $n \rightarrow \infty$ based on the non-backtracking (Hashimoto) operator. Following the same idea in [6], Coste proved the spectral gap for $d$-regular digraphs [14] and Brito et al. [9] proved an analog of Alon's spectral gap conjecture for random bipartite biregular graphs; for deterministic ones, the equivalent of the Alon-Boppana bound had first been shown by Lin and Solé [26].

It is thus fair to say that both graph expansion and the spectral gap in regular graphs and quasi-regular graphs are now very well understood; by contrast, despite the natural applications and extension possibilities, hypergraph expansion is a much less understood area. The difficulty here is that it is not immediately clear which operator or structure to associate to the hypergraph. There are three main takes on this: the Feng-Li approach [16], which defined an adjacency matrix, the Friedman-Wigderson tensor approach [18], and the Lu-Peng approach [30,31], which defined a sequence of Laplacian matrices through higher-order random walks.

Several results on hypergraph expansion have been obtained using the FriedmanWigderson approach. Hyperedge expansion depending on the spectral norm of the associated tensor was studied in the original paper [18], the relation between the spectral gap and quasirandom properties was discussed in Lenz and Mubayi [22, 23], and an inverse expander mixing lemma was obtained in Cohen et al. [12]. Very recently, Li and Mohar [24] proved a generalization of the Alon-Boppana bound to $(d, k)$-regular hypergraphs for their adjacency tensors. On the other side, using the Feng-Li adjacency matrix approach, the original paper [16] proved the Alon-Boppana lower bound for the adjacency matrix of regular hypergraphs, and then Li and Solé [26] defined a $(d, k)$-regular hypergraph to 
be Ramanujan if any eigenvalue $\lambda \neq d(k-1)$ satisfies

$$
|\lambda-k+2| \leqslant 2 \sqrt{(d-1)(k-1)} .
$$

Ramanujan hypergraphs were further studied in $[33,25,37]$. Note that when $k=2$ (when the hypergraphs are actual graphs), this definition coincides with the definition for Ramanujan graphs. The adjacency matrices and Laplacian matrices of general uniform hypergraphs were analyzed in [4], where the relation between eigenvalues and diameters, random walks, Ricci curvature of the hypergraphs were studied.

In this paper, we fill in the gaps in the literature by showing a spectral gap for the adjacency matrix of a hypergraph, following the Feng-Li definition; we connect it to the mixing rate of the hypergraph random walk considered in [45] and subsequently studied in $[13,20]$, and we also show that this gap governs hyperedge and vertex expansion of the hypergraph, thus completing the parallel with graph results. Specifically, for $(d, k)$-regular hypergraphs and their adjacency matrices (the precise definitions are given in the next section), we prove the following:

- Hyperedge and vertex expansion are controlled by the second eigenvalue of the adjacency matrix.

- The mixing rate of the random walk is controlled by the second eigenvalue of the adjacency matrix.

- The uniformly random $(d, k)$-regular hypergraph model has a spectral gap. This is by far the most exciting result, and it turns out to be a simple consequence of the spectral gap of uniformly random bipartite biregular graphs [9]. Our result shows that, asymptotically, almost all $(d, k)$-regular hypergraphs are almost Ramanujan in the sense of Li-Solé (see (1)).

Other results include the spectral gap and description for the spectrum of the nonbacktracking operator of the hypergraph, the limiting empirical distribution for the spectrum of the adjacency matrix of the uniformly random $(d, k)$-regular hypergraph in different regimes (which was studied by Feng and Li in [16] for deterministic sequences of hypergraphs with few cycles and fixed $d, k)$, and a sort of local law of this empirical spectral distribution.

Our main methodology is to translate the results from bipartite biregular graphs by using the bijection between the spectra (Lemma 10). While this bijection has been known for a long time, the results on bipartite biregular graphs [15, 9] (especially the spectral gap) are quite recent.

Our spectral gap results are linked to the random walk and offer better control over the mixing rate. Together with the Alon-Boppana result established by Feng-Li [16], they give complete control over the behavior of the random walk and hyperedge/vertex expansion. In our view, this establishes the adjacency matrix perspective of Feng and $\mathrm{Li}$ as ultimately more useful not just theoretically, but possibly computationally as well, since computing second eigenvalues of matrices is achievable in polynomial time, whereas the complexity of computing spectral norms of tensors is NP-hard [21]. 
The rest of the paper is structured as follows. In Section 2 we provide definitions and properties of hypergraphs that we use in the paper. In Section 3 we show that several expansion properties of $(d, k)$-regular hypergraphs are related to the second eigenvalues of their adjacency matrices. In Section 4 we prove the analog of Friedman's second eigenvalue theorem for uniformly random $(d, k)$-regular hypergraphs. The spectra of the non-backtracking operator for the hypergraph are analyzed in Section 5. Finally, we study the empirical spectral distributions of uniformly random $(d, k)$-regular hypergraphs in Section 6.

\section{Preliminaries}

Definition 1 (hypergraph). A hypergraph $H$ consists of a set $V$ of vertices and a set $E$ of hyperedges such that each hyperedge is a nonempty subset of $V$. A hypergraph $H$ is $k$-uniform for an integer $k \geqslant 2$ if every hyperedge $e \in E$ contains exactly $k$ vertices. The degree of $i$, denoted $\operatorname{deg}(i)$, is the number of all hyperedges incident to $i$. A hypergraph is $d$-regular if all of its vertices have degree $d$. A hypergraph is $(d, k)$-regular if it is both $d$-regular and $k$-uniform. A vertex $i$ is incident to a hyperedge $e$ if and only if $v$ is an element of $e$. We can define the incidence matrix $X$ of a hypergraph to be a $|V| \times|E|$ matrix indexed by elements in $V$ and $E$ such that $X_{i, e}=1$ if $i \in e$ and 0 otherwise. Moreover, if we regard $X$ as the adjacency matrix of a graph, it defines a bipartite graph $G$ with two vertex sets being $V$ and $E$. We call $G$ the bipartite graph associated to $H$.

Definition 2 (walks and cycles). A walk of length $l$ on a hypergraph $H$ is a sequence $\left(v_{0}, e_{1}, v_{1}, \cdots, e_{l}, v_{l}\right)$ such that $v_{j-1} \neq v_{j}$ and $\left\{v_{j-1}, v_{j}\right\} \subset e_{j}$ for all $1 \leqslant j \leqslant l$. A walk is closed if $v_{0}=v_{l}$. A cycle of length $l$ in a hypergraph $H$ is a closed walk $\left(v_{0}, e_{1}, \ldots, v_{l-1}, e_{l}, v_{l+1}\right)$ such that

- $\left|\left\{e_{1}, \ldots, e_{l}\right\}\right|=l$ (all hyperedges are distinct);

- $\left|\left\{v_{0}, \ldots v_{l-1}\right\}\right|=l, v_{l+1}=v_{0}$ (all vertices are distinct subject to $v_{l+1}=v_{0}$ ).

In the associated bipartite graph $G$, a cycle of length $l$ in $H$ corresponds to a cycle of length $2 l$. We say $H$ is connected if for any $i, j \in V$, there is a walk between $i, j$. It's easy to see $H$ is connected if and only if the corresponding bipartite graph $G$ is connected.

Definition 3 (adjacency matrix). For a hypergraph $H$ with $n$ vertices, we associate a $n \times n$ symmetric matrix $A$ called the adjacency matrix of $H$. For $i \neq j, A_{i j}$ is the number of hyperedges containing both $i$ and $j$ and $A_{i i}=0$ for all $1 \leqslant i \leqslant n$.

If $H$ is 2-uniform, this is the adjacency matrix of an ordinary graph. The largest eigenvalue of $A$ for $(d, k)$-regular hypergraphs is $d(k-1)$ with eigenvector $\frac{1}{\sqrt{n}}(1, \ldots, 1)$.

\section{$3 \quad$ Expansion and mixing properties of regular hypergraphs}

In this section, we relate the expansion property of a regular hypergraph to its second eigenvalue. We prove results on expander mixing and vertex expansion, and compute the 
mixing rate of simple random walks and non-backtracking random walks. These results follow easily from the same methodology used in Chung's book [10]. Let $H=(V, E)$ be a $(d, k)$-regular hypergraph, for any subsets $V_{1}, V_{2} \subset V$, define

$$
e\left(V_{1}, V_{2}\right):=\left|\left\{(i, j, e): i \in V_{1}, j \in V_{2},\{i, j\} \in e \subset E\right\}\right|
$$

which counts the number of hyperedges between vertex set $V_{1}, V_{2}$ with multiplicity. For each hyperedge $e$, the multiplicity is given by $\left|e \cap V_{1}\right| \cdot\left|e \cap V_{2}\right|$. We first provide an edge mixing result whose equivalence for regular graphs is given in [10].

Theorem 4 (expander mixing). Let $H=(V, E)$ be a $(d, k)$-regular hypergraph with adjacency matrix $A$. Let $\lambda=\max \left\{\lambda_{2}(A),\left|\lambda_{n}(A)\right|\right\}$. The following holds: for any subsets $V_{1}, V_{2} \subset V$,

$$
\left|e\left(V_{1}, V_{2}\right)-\frac{d(k-1)}{n}\right| V_{1}|\cdot| V_{2}|| \leqslant \lambda \sqrt{\left|V_{1}\right| \cdot\left|V_{2}\right|\left(1-\frac{\left|V_{1}\right|}{n}\right)\left(1-\frac{\left|V_{2}\right|}{n}\right)} .
$$

Remark 5. The above result is qualitatively different from the expander mixing lemma for $k$-uniform regular graphs studied in $[18,12]$. Their result considers the number of hyperedges between any $k$ subsets of $V$ and the parameter $\lambda$ there is the spectral norm of a tensor associated with the hypergraph.

Proof. Let $1_{V_{i}}$ be the indicator vector of the set $V_{i}$ for $i=1,2$. Let $v_{1}, \ldots, v_{n}$ be the unit eigenvector associated to $\lambda_{1}, \ldots \lambda_{n}$ of $A$. We have the following decomposition of $1_{V_{1}}, 1_{V_{2}}$ :

$$
1_{V_{1}}=\sum_{i=1}^{n} \alpha_{i} v_{i}, \quad 1_{V_{2}}=\sum_{i=1}^{n} \beta_{i} v_{i}
$$

for some numbers $\alpha_{i}, \beta_{i}, 1 \leqslant i \leqslant n$. Recall that $\lambda_{1}=d(k-1)$ and $v_{1}=\frac{1}{\sqrt{n}}(1, \ldots 1)^{\top}$. We have $\alpha_{1}=\left\langle 1_{V_{1}}, v_{1}\right\rangle=\frac{\left|V_{1}\right|}{\sqrt{n}}, \beta_{1}=\left\langle 1_{V_{2}}, v_{1}\right\rangle=\frac{\left|V_{2}\right|}{\sqrt{n}}$. From the definition of $e\left(V_{1}, V_{2}\right)$,

$$
\begin{aligned}
e\left(V_{1}, V_{2}\right) & =\sum_{1 \leqslant i, j \leqslant n} 1_{V_{1}}(i) 1_{V_{2}}(j) A_{i j}=\left\langle 1_{V_{1}}, A 1_{V_{2}}\right\rangle \\
& =\lambda_{1} \alpha_{1} \beta_{1}+\sum_{i \geqslant 2} \lambda_{i} \alpha_{i} \beta_{i}=\frac{d(k-1)}{n}\left|V_{1}\right| \cdot\left|V_{2}\right|+\sum_{i \geqslant 2} \lambda_{i} \alpha_{i} \beta_{i} .
\end{aligned}
$$

Therefore by the Cauchy-Schwarz inequality,

$$
\left|e\left(V_{1}, V_{2}\right)-\frac{d(k-1)}{n}\right| V_{1}|\cdot| V_{2}|| \leqslant \lambda \sum_{i \geqslant 2}\left|\alpha_{i} \beta_{i}\right| \leqslant \lambda \sqrt{\sum_{i \geqslant 2} \alpha_{i}^{2}} \sqrt{\sum_{i \geqslant 2} \beta_{i}^{2}} .
$$

Note that

$$
\sum_{i \geqslant 2} \alpha_{i}^{2}=\sum_{i \geqslant 1} \alpha_{i}^{2}-\frac{\left|V_{1}\right|^{2}}{n}=\left|V_{1}\right|\left(1-\frac{\left|V_{1}\right|}{n}\right)
$$


and similarly, $\sum_{i \geqslant 2} \beta_{i}^{2}=\left|V_{2}\right|\left(1-\frac{\left|V_{2}\right|}{n}\right)$. This implies

$$
\left|e\left(V_{1}, V_{2}\right)-\frac{d(k-1)}{n}\right| V_{1}|\cdot| V_{2}|| \leqslant \lambda \sum_{i \geqslant 2}\left|\alpha_{i} \beta_{i}\right| \leqslant \lambda \sqrt{\left|V_{1}\right| \cdot\left|V_{2}\right|\left(1-\frac{\left|V_{1}\right|}{n}\right)\left(1-\frac{\left|V_{2}\right|}{n}\right)} .
$$

For any subset $S \subset V$, we define its neighborhood set to be

$$
N(S):=\{i \text { : there exists } j \in S \text { such that }\{i, j\} \subset e \text { for some } e \in E\} .
$$

We have the following result on vertex expansion of regular hypergraphs.

Theorem 6 (vertex expansion). Let $H=(V, E)$ be a $(d, k)$-regular hypergraph with adjacency matrix $A$. Let $\lambda=\max \left\{\lambda_{2}(A),\left|\lambda_{n}(A)\right|\right\}$. For any subset $S \subset V$, we have that

$$
\frac{|N(S)|}{|S|} \geqslant \frac{1}{1-\left(1-\frac{\lambda^{2}}{d^{2}(k-1)^{2}}\right)\left(1-\frac{|S|}{n}\right)} \text {. }
$$

Proof. Let $1_{S}$ be the indicator vector of the set $S$ with the decomposition $1_{S}=\sum_{i=1}^{n} \gamma_{i} v_{i}$, where $\gamma_{i}, 1 \leqslant i \leqslant n$ are constants and $v_{i}, 1 \leqslant i \leqslant n$ are the unit eigenvectors of $A$ associated to $\lambda_{1}, \ldots, \lambda_{n}$, respectively. Then we know $\gamma_{1}=\frac{|S|}{\sqrt{n}}$ and

$$
\begin{aligned}
\left\|A 1_{S}\right\|_{2}^{2} & =\sum_{i=1}^{n} \lambda_{i}^{2} \gamma_{i}^{2} \leqslant d^{2}(k-1)^{2} \frac{|S|^{2}}{n}+\lambda^{2}\left(\sum_{i \geqslant 2} \gamma_{i}^{2}\right) \\
& =d^{2}(k-1)^{2} \frac{|S|^{2}}{n}+\lambda^{2}\left(|S|-\frac{|S|^{2}}{n^{2}}\right)=\left(d^{2}(k-1)^{2}-\lambda^{2}\right) \frac{|S|^{2}}{n}+\lambda^{2}|S| .
\end{aligned}
$$

On the other hand,

$$
\left\|A 1_{S}\right\|_{2}^{2}=\left\langle A 1_{S}, A 1_{S}\right\rangle=\sum_{i=1}^{n}\left(\sum_{k \in S} A_{i k}\right)^{2}=\sum_{i=1}^{n} e(\{i\}, S)^{2}=\sum_{i \in N(S)} e(\{i\}, S)^{2},
$$

and by Cauchy-Schwartz inequality,

$$
\sum_{i \in N(S)} e(\{i\}, S)^{2} \geqslant \frac{\left(\sum_{i \in N(S)} e(S,\{i\})\right)^{2}}{|N(S)|}
$$

The quantity

$$
\sum_{i \in N(S)} e(S,\{i\})=e(S, N(S))=|\{(i, j, e): i \in S, j \in N(S),\{i, j\} \subset e \in E\}|
$$

counts the number of hyperedges between $S$ and $N(S)$ with multiplicity. We then have

$$
e(S, N(S))=|S|(k-1) d .
$$

Putting Equations (3)-(7) together, we obtain (2). 
For the rest of this section, we compute the mixing rates of random walks on hypergraphs. The simple random walk on a general hypergraph was first defined in [45], where the authors gave a random walk explanation of the spectral methods for clustering and segmentation on hypergraphs, which generalized the result in Meila and Shi [36] for graphs. A quantum version of random walks on regular hypergraphs was recently studied by Liu et al. [28].

The simple random walk on $k$-uniform hypergraphs has the following transition rule. Start at a vertex $v_{0}$. If at the $t$-th step we are at vertex $v_{t}$, we first choose a hyperedge $e$ uniformly over all hyperedges incident with $v_{t}$, and then choose a vertex $v_{t+1} \in e, v_{t+1} \neq v_{t}$ uniformly at random. The sequence of random vertices $\left(v_{t}, t \geqslant 0\right)$ is a Markov chain. It generalizes the simple random walk on graphs. We denote by $P=\left(P_{i j}\right)_{1 \leqslant i, j \leqslant n}$ the transition matrix for the Markov chain and let $D$ be the diagonal matrix with $D_{i i}=$ $\operatorname{deg}(i), 1 \leqslant i \leqslant n$. From the definition of the simple random walk on hypergraphs, for any $(d, k)$-regular hypergraphs with adjacency matrix $A$, the transition matrix satisfies $P=\frac{1}{d(k-1)} A$.

It's known (see for example [29]) that for any graph (or multigraph) $G$, if $G$ is connected and non-bipartite, then it has a unique stationary distribution. For $d$-regular graphs, being connected and non-bipartite is equivalent to requiring $\lambda=\max \left\{\lambda_{2}(A),\left|\lambda_{n}(A)\right|\right\}<d$, see for example [2]. The simple random walk on $(d, k)$-regular hypergraphs $H=(V, E)$ can also be seen as a simple random walk on a multigraph $G_{H}$ on $V$, where the number of edges between $i, j$ in $G_{H}$ is $A_{i j}$. The adjacency matrix of $G_{H}$ is the same as the adjacency matrix of $H$. Therefore the simple random walk on $H$ converges to a unique stationary distribution if and only if the multigraph $G_{H}$ is connected and non-bipartite. These two conditions can be satisfied as long as we have the following condition on the second eigenvalue.

Lemma 7 . Let $H$ be a $(d, k)$-regular hypergraph with adjacency matrix $A$. The simple random walk on $H$ converges to a stationary distribution if and only if

$$
\lambda=\max \left\{\lambda_{2}(A),\left|\lambda_{n}(A)\right|\right\}<d(k-1) .
$$

Proof. If the corresponding multigraph $G_{H}$ is bipartite, then we have $\lambda_{n}=-\lambda_{1}=$ $-d(k-1)$. If $G_{H}$ is not connected, then it has at least two connected components, the largest eigenvalue will have multiplicity $\geqslant 2$, which implies $\lambda=d(k-1)$. Therefore $\lambda<d(k-1)$ if and only if $G_{H}$ is non-bipartite and connected. From the general theory of Markov chains on graphs and multigraphs, the simple random walk on $G_{H}$ converges to a stationary distribution. Therefore the simple random walk on $H$ converges to a stationary distribution.

For any $(d, k)$-regular hypergraph $H$ with $\lambda<d(k-1)$, a simple calculation shows that the stationary distribution is $\pi(i)=\frac{1}{n}$ for all $i \in V$. The mixing rate of the simple random walk on hypergraphs, which measures how fast the Markov chain converges to the stationary distribution, is defined by

$$
\rho(H):=\limsup _{l \rightarrow \infty} \max _{i, j \in V}\left|\left(P^{l}\right)_{i j}-\pi(j)\right|^{1 / l},
$$


where $\pi$ is the unique stationary distribution on $V$. Let $\lambda_{1} \geqslant \lambda_{2} \geqslant \cdots \geqslant \lambda_{n}$ be the eigenvalues of $A$ and we define the second eigenvalue in absolute value of $A$ by $\lambda:=$ $\max \left\{\lambda_{2},\left|\lambda_{n}\right|\right\}$.

The non-backtracking walk on hypergraphs is defined in [38] as a generalization of non-backtracking walk on graphs. Recall a walk of length $l$ in a hypergraph is a sequence $w=\left(v_{0}, e_{1}, v_{1}, e_{2}, \ldots v_{l-1}, e_{l}, v_{l}\right)$ such that $v_{i} \neq v_{i+1}$ and $\left\{v_{i}, v_{i+1}\right\} \subset e_{i+1}$ for all $0 \leqslant i \leqslant$ $l-1$. We say $w$ is a non-backtracking walk if $e_{i} \neq e_{i+1}$ for $1 \leqslant i \leqslant l-1$. Define a non-backtracking random walk of length $l$ on $H$ from some given vertex $v_{0} \in V$, to be a uniformly chosen member of the set of non-backtracking walks of length $l$ starting at $v_{0}$. Let

$$
\vec{E}(H):=\{(i, e): i \in V(H), e \in E(H), i \in e\}
$$

be the set of oriented hyperedges of a $k$-uniform hypergraph $H$. Similar to case for regular graphs in [2], we can also consider the non-backtracking random walk on $H$ starting from an initial vertex $v_{0}$ as a Markov chain $\left\{X_{t}\right\}_{t \geqslant 0}$ with a state space $\vec{E}(H)$ in the following way. The distribution of the initial state is given by

$$
\mathbb{P}\left(X_{0}=\left(v_{0}, e\right)\right)=\frac{\mathbf{1}_{v_{0} \in e}}{\operatorname{deg}\left(v_{0}\right)},
$$

for any $e \in E(H)$. The transition probability is given by

$$
\begin{aligned}
& \mathbb{P}\left(X_{t+1}=(u, f) \mid X_{t}=(v, e)\right) \\
= & \begin{cases}\frac{1}{(k-1)(\operatorname{deg}(u)-1)} & \text { if } u \in e, u \neq v \in V(H), \text { and } f \neq e \in E(H), \\
0 & \text { otherwise. }\end{cases}
\end{aligned}
$$

Notice that if $H$ is a $(d, k)$-regular hypergraph with $(d, k)=(2,2)$, then $H$ is a 2 regular graph, which is a disjoint union of cycles. The non-backtracking random walk on $H$ is periodic and does not converge to a stationary distribution. Given a $(d, k)$-regular hypergraph $H=(V, E)$ with $(d, k) \neq(2,2)$, let $\tilde{P}_{i, j}^{(l)}$ be the transition probability that a non-backtracking random walk of length $l$ on $H$ starts at $i$ and ends at $j$. Define

$$
\tilde{\rho}(H):=\limsup _{l \rightarrow \infty} \max _{i, j \in V}\left|\tilde{P}_{i j}^{(l)}-\frac{1}{n}\right|^{1 / l}
$$

to be the mixing rate of the non-backtracking random walk. As a generalization of the result in [2], we can connect the second eigenvalue of regular hypergraphs to the mixing rate of non-backtracking random walk. It turns out that similar results were already studied in [11] for clique-wise non-backtracking walks on regular graphs. Especially, Corollary 1.3 in [11] is equivalent to the following theorem. We include a proof here for completeness.

Theorem 8. Let $H$ be $a(d, k)$-regular hypergraph with $d, k \geqslant 2$ whose adjacency matrix has the second largest eigenvalue in absolute value $\lambda:=\max \left\{\lambda_{2},\left|\lambda_{n}\right|\right\}<d(k-1)$, then 
1. the mixing rate of the simple random walk on $H$ is $\rho(H)=\frac{\lambda}{(k-1) d}$.

2. Assume further that $(d, k) \neq(2,2)$. Define a function $\psi:[0, \infty) \rightarrow \mathbb{R}$ as

$$
\psi(x):= \begin{cases}x+\sqrt{x^{2}-1} & \text { if } x \geqslant 1 \\ 1 & \text { if } 0 \leqslant x \leqslant 1 .\end{cases}
$$

Then a non-backtracking random walk on $H$ converges to the uniform distribution, and its mixing rate $\tilde{\rho}(H)$ satisfies $\tilde{\rho}(H)=\frac{1}{\sqrt{(d-1)(k-1)}} \psi\left(\frac{\lambda}{2 \sqrt{(k-1)(d-1)}}\right)$.

Proof. (1) We first consider simple random walks. For any $l \geqslant 1, P^{l}=\frac{1}{((k-1) d)^{l}} A^{l}$ and the vector $v_{1}=\frac{1}{\sqrt{n}}(1, \ldots, 1)$ is an eigenvector of $P^{l}$ corresponding to the unique largest eigenvalue 1 . Let $\mu(l)=\max \left\{\left|\lambda_{2}\left(P^{l}\right)\right|,\left|\lambda_{n}\left(P^{l}\right)\right|\right\}$, we have

$$
\max _{i j}\left|P_{i j}^{l}-\frac{1}{n}\right|=\max _{i j}\left|\left\langle\left(P^{l}-v_{1}^{\top} v_{1}\right) e_{i}, e_{j}\right\rangle\right| \leqslant \max _{|u|=|v|=1}\left|\left\langle\left(P^{l}-v_{1}^{\top} v_{1}\right) u, v\right\rangle\right|=\mu(l)=\frac{\lambda^{l}}{(k-1)^{l} d^{l}} .
$$

This implies $\rho(H) \leqslant \frac{\lambda}{(k-1) d}$. On the other hand, let $J$ be a $n \times n$ matrix whose entries are all 1 , we have

$$
\begin{aligned}
\max _{i j}\left|P_{i j}^{l}-\frac{1}{n}\right| & \geqslant \frac{1}{n} \sqrt{\sum_{i j}\left|\left(P^{l}\right)_{i j}-\frac{1}{n}\right|^{2}}=\frac{1}{n}\left\|P^{l}-\frac{1}{n} J\right\|_{F}=\frac{1}{n} \sqrt{\sum_{2 \leqslant i \leqslant n} \lambda_{i}^{2}\left(P^{l}\right)} \\
& \geqslant \frac{\mu(l)}{n}=\frac{1}{n} \frac{\lambda^{l}}{(k-1)^{l} d^{l}}
\end{aligned}
$$

which implies $\rho(H) \geqslant \frac{\lambda}{(k-1) d}$. This completes the proof of part (1) of Theorem 8 .

For part (2), we follow the steps in [2]. Recall that the Chebyshev polynomials satisfy the following recurrence relation: $U_{k+1}(x)=2 x U_{k}(x)-U_{k-1}(x), \forall k \geqslant 0$. We also define $U_{-1}(x)=0, U_{0}(x)=1$. Let $A$ be the adjacency matrix of $H$ and define the matrix $A^{(l)}$ such that $A_{i j}^{(l)}$ is the number of non-backtracking walks of length $l$ from $i$ to $j$ for all $i, j$. By definition, the matrices $A^{(l)}$ satisfy the following recurrence:

$$
\left\{\begin{array}{l}
A^{(1)}=A, \quad A^{(2)}=A^{2}-(k-1) d I, \\
A^{(l+1)}=A A^{(l)}-(k-1)(d-1) A^{(l-1)} \text { for } l \geqslant 2,
\end{array}\right.
$$

where $(k-1) d I$ in the first equation eliminates the diagonal of $A^{2}$ to avoid backtracking, and $(k-1)(d-1) A^{(l-1)}$ in the second equation of $(9)$ eliminates the walk which backtracks in the $(l+1)$-st step. We claim that

$$
A^{(l)}=\sqrt{(k-1)^{l} d(d-1)^{l-1}} Q_{l}\left(\frac{A}{2 \sqrt{(k-1)(d-1)}}\right),
$$


where $Q_{l}(x)=\sqrt{\frac{d-1}{d}} U_{l}(x)-\frac{1}{\sqrt{d(d-1)}} U_{l-2}(x)$ for all $l \geqslant 1$. To see this, let

$$
f(A, l):=\sqrt{(k-1)^{l} d(d-1)^{l-1}} Q_{l}\left(\frac{A}{2 \sqrt{(k-1)(d-1)}}\right) .
$$

Since $U_{1}(x)=2 x, U_{2}(x)=4 x^{2}-1$, we have

$$
Q_{1}(x)=2 \sqrt{\frac{d-1}{d}} x, \quad Q_{2}(x)=\sqrt{\frac{d-1}{d}}\left(4 x^{2}-1\right)-\frac{1}{\sqrt{d(d-1)}} .
$$

We can check that

$$
\begin{aligned}
& f(A, 1)=\sqrt{(k-1) d} \cdot Q_{1}\left(\frac{A}{2 \sqrt{(k-1)(d-1)}}\right)=A=A^{(1)}, \\
& f(A, 2)=\sqrt{(k-1)^{2} d(d-1)} \cdot Q_{2}\left(\frac{A}{2 \sqrt{(k-1)(d-1)}}\right)=A^{2}-(k-1) d I=A^{(2)} .
\end{aligned}
$$

Therefore (10) holds for $l=1,2$. Since $Q_{l}(x)$ is a linear combination of $U_{l-2}, U_{l}$, it satisfies the recurrence $Q_{k+1}(x)=2 x Q_{k}(x)-Q_{k-1}(x)$. Therefore by induction we have $f(A, l)=A^{(l)}$ for all $l \geqslant 1$. Recall $\tilde{P}_{i, j}^{(l)}$ is the probability that a non-backtracking random walk of length $l$ on $H$ starts from $i$ and ends in $j$. The number of all possible nonbacktracking walks of length $l$ starting from $i$ is $d(k-1)((k-1)(d-1))^{l-1}$. This is because for the first step we have $d(k-1)$ many choices for hyperedges and vertices, and for the remaining $(l-1)$ steps we have $((k-1)(d-1))^{l-1}$ many choices in total. Normalizing $A^{(l)}$ yields

$$
\tilde{P}_{i j}^{(l)}=\frac{A_{i j}^{(l)}}{(k-1) d((k-1)(d-1))^{l-1}}=\frac{A_{i j}^{(l)}}{d(d-1)^{l-1}(k-1)^{l}} .
$$

Let $\tilde{\mu}_{1}(l)=1, \tilde{\mu}_{2}(l) \geqslant \cdots \geqslant \tilde{\mu}_{n}(l)$ be the eigenvalues of $\tilde{P}^{(l)}, \tilde{\mu}(l):=\max \left\{\left|\mu_{2}(l)\right|,\left|\mu_{n}(l)\right|\right\}$. We obtain that $\tilde{P}^{(l)}$ is precisely the transition matrix of a non-backtracking random walk of length $l$. Same as Claim 2.2 in [2], we have

$$
\frac{\tilde{\mu}(l)}{n} \leqslant \max _{i j}\left|\tilde{P}_{i j}^{(l)}-\frac{1}{n}\right| \leqslant \tilde{\mu}(l) .
$$

We sketch the proof of $(12)$ here. Since $\tilde{P}^{(l)}$ is doubly stochastic, $v_{1}=\frac{1}{\sqrt{n}}(1, \ldots, 1)$ is an eigenvector of $\tilde{P}^{(l)}$ corresponding to the largest eigenvalue 1 . We have

$$
\max _{i j}\left|\tilde{P}_{i j}^{(l)}-\frac{1}{n}\right|=\max _{i j}\left|\left\langle\left(\tilde{P}^{(l)}-v_{1}^{\top} v_{1}\right) e_{i}, e_{j}\right\rangle\right| \leqslant \max _{\|u\|_{2}=\|v\|_{2}=1}\left|\left\langle\left(\tilde{P}^{(l)}-v_{1}^{\top} v_{1}\right) u, v\right\rangle\right|=\tilde{\mu}(l) .
$$

On the other hand, let $J$ be as above, we have

$$
\max _{i j}\left|\tilde{P}_{i j}^{(l)}-\frac{1}{n}\right| \geqslant \frac{1}{n} \sqrt{\sum_{i j}\left|\tilde{P}_{i j}^{(l)}-\frac{1}{n}\right|^{2}}=\frac{1}{n}\left\|\tilde{P}^{(l)}-\frac{1}{n} J\right\|_{F}=\frac{1}{n} \sqrt{\sum_{2 \leqslant i \leqslant n} \lambda_{i}^{2}\left(\tilde{P}^{(l)}\right)} \geqslant \frac{\tilde{\mu}(l)}{n} .
$$


Therefore

$$
\tilde{\rho}(H)=\limsup _{l \rightarrow \infty} \max _{i, j \in V}\left|\tilde{P}_{i j}^{(l)}-\frac{1}{n}\right|^{1 / l}=\limsup _{l \rightarrow \infty} \tilde{\mu}(l)^{1 / l} .
$$

By $(10)$ and (11), for $1 \leqslant i \leqslant n$,

$$
\tilde{\mu}_{i}(l)=\frac{\lambda_{i}\left(A^{(l)}\right)}{d(d-1)^{l-1}(k-1)^{l}}=\frac{1}{\sqrt{d(d-1)^{l-1}(k-1)^{l}}} Q_{l}\left(\frac{\lambda_{i}(A)}{2 \sqrt{(k-1)(d-1)}}\right) .
$$

From Lemma 2.3. in [2],

$$
\limsup _{l \rightarrow \infty}\left|Q_{l}(x)\right|^{1 / l}=\psi(|x|)= \begin{cases}1 & 0 \leqslant x \leqslant 1 \\ |x|+\sqrt{x^{2}-1} & x \in \mathbb{R} \backslash[-1,1] .\end{cases}
$$

Therefore $\tilde{\rho}(H)=\frac{1}{\sqrt{(d-1)(k-1)}} \psi\left(\frac{\lambda}{2 \sqrt{(k-1)(d-1)}}\right)$. This completes the proof.

\section{Spectral gap of random regular hypergraphs}

Let $\mathcal{G}\left(n, m, d_{1}, d_{2}\right)$ be the set of all simple bipartite biregular random graphs with vertex set $V=V_{1} \cup V_{2}$ such that $\left|V_{1}\right|=n,\left|V_{2}\right|=m$, and every vertex in $V_{i}$ has degree $d_{i}$ for $i=1,2$. Here we must have $n d_{1}=m d_{2}=|E|$. Let $\mathcal{H}(n, d, k)$ be the set of all simple (without multiple hyperedges) $(d, k)$-regular hypergraphs with labelled vertex set $[n]$ and $\frac{n d}{k}$ many labelled hyperedges denoted by $\left\{e_{1}, \ldots, e_{n d / k}\right\}$.

Remark 9 . From this section on, we always assume $d \geqslant k$ for simplicity, since a $(d, k)$ regular hypergraph, its dual hypergraph is $(k, d)$-regular, and they have the same associated bipartite biregular graph by swapping the vertex sets $V_{1}$ and $V_{2}$.

It's well known (see for example [16]) that there exists a bijection between regular multi-hypergraphs and bipartite biregular graphs. See Figure 1 as an example of the bijection. For a given bipartite biregular graph, if there are two vertices in $V_{2}$ that share the same set of neighbors in $V_{1}$, the corresponding regular hypergraph will have multiple hyperedges, see Figure 2. Let $\mathcal{G}^{\prime}\left(n, m, d_{1}, d_{2}\right)$ be a subset of $\mathcal{G}\left(n, m, d_{1}, d_{2}\right)$ such that for any $G \in \mathcal{G}^{\prime}\left(n, m, d_{1}, d_{2}\right)$, the vertices in $V_{2}$ have different sets of neighborhoods in $V_{1}$. We obtain the following bijection.

Lemma 10. There is a bijection between the set $\mathcal{H}(n, d, k)$ and the set $\mathcal{G}^{\prime}(n, n d / k, d, k)$.

Proof. Let $G \in \mathcal{G}^{\prime}(n, n d / k, d, k)$ be an $(n, n d / k, d, k)$-bipartite biregular graph, and $A_{G}$ be its adjacency matrix, we then have $A_{G}=\left(\begin{array}{cc}0 & X \\ X^{\top} & 0\end{array}\right)$, where $X$ is a $n \times(n d / k)$ matrix with entries $X_{i j}=1$ if and only there is an edge between $i \in V_{1}, j \in V_{2}$. We can then construct a regular hypergraph $H=(V(H), E(H))$ from $X$ with $V(H)=V_{1}$. There exists an edge $e_{j}=\left\{i_{1}, \ldots, i_{k}\right\} \in E(H)$ if and only if $j \in V_{2}$ and $i_{1}, \ldots, i_{k} \in V_{1}$ are connected to $j$ in $G$. By the definition of $G$, vertices in $V_{2}$ have different sets of neighbors, hence the 

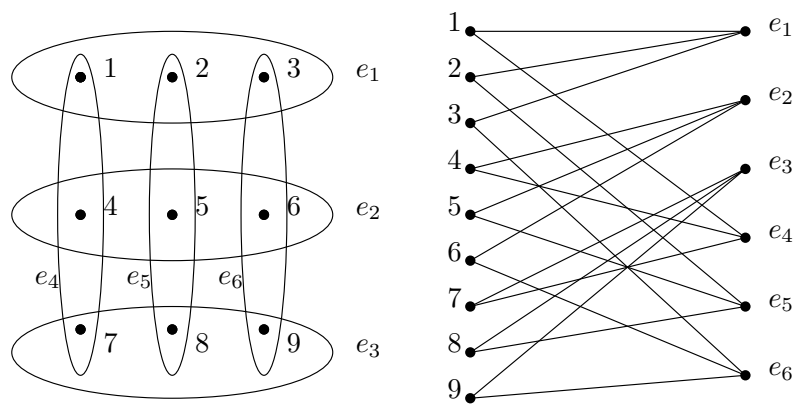

Figure 1: a $(2,3)$-regular hypergraph and its associated bipartite biregular graph where all vertices in $V_{2}$ have different neighborhoods in $V_{1}$

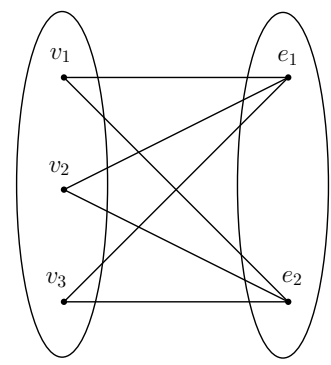

Figure 2: a subgraph in a bipartite biregular graph which gives multiple hyperedges $e_{1}$ and $e_{2}$ in the corresponding regular hypergraph

corresponding hypergraph $H$ has no multiple hyperedges. It's easy to check that $H$ is a $(d, k)$-regular hypergraph on $n$ vertices.

Conversely, for any simple $(d, k)$-regular hypergraph $H \in \mathcal{H}(n, d, k), X$ corresponds the incidence matrix of $H$, and we can associate to $H$ a $(n, n d / k, d, k)$-bipartite biregular graph $G$ whose adjacency matrix is $\left(\begin{array}{cc}0 & X \\ X^{\top} & 0\end{array}\right)$, and it has no two vertices in $V_{2}$ sharing the same set of neighbors.

From Lemma 10, the uniform distribution on $\mathcal{G}^{\prime}(n, n d / k, d, k)$ for bipartite biregular graphs induces the uniform distribution on $\mathcal{H}(n, d, k)$ for regular hypergraphs. With this observation, we are able to translate the results for spectra of random bipartite biregular graphs into results for spectra of random regular hypergraphs. Our first step is the following spectral gap result.

Theorem 11. Let $A$ be the adjacency matrix of a random $(d, k)$-regular hypergraph sampled uniformly from $\mathcal{H}(n, d, k)$ with $d \geqslant k \geqslant 3$, then any eigenvalue $\lambda(A) \neq d(k-1)$ satisfies

$$
|\lambda(A)-k+2| \leqslant 2 \sqrt{(k-1)(d-1)}+\epsilon_{n},
$$

asymptotically almost surely with $\epsilon_{n} \rightarrow 0$ as $n \rightarrow \infty$.

Remark 12. For $k=2$, Theorem 11 reduces to Alon's second eigenvalue conjecture proved 
in $[17,6]$. In terms of Ramanujan hypergraphs defined in (1), the theorem implies almost every $(d, k)$-regular hypergraph is almost Ramanujan.

We start with the following lemma connecting the adjacency matrix of a regular hypergraph and its associated bipartite biregular graph.

Lemma 13. Let $H$ be a $(d, k)$-regular hypergraph, and let $G$ be the corresponding bipartite biregular graph associated to $H$. Let $A_{H}$ be the adjacency matrix of $H$, and $A_{G}$ be the adjacency matrix of $G$ with the form

$$
A_{G}=\left(\begin{array}{cc}
0 & X \\
X^{\top} & 0
\end{array}\right)
$$

Then $X X^{\top}=A_{H}+d I$.

Proof. Let $V$ and $E$ be the vertex and hyperedge set of $H$ respectively. For $i \neq j$, we have

$$
\left(X X^{\top}\right)_{i j}=\sum_{e \in E} X_{i e} X_{j e}=\sum_{e \in E} \mathbf{1}_{\{i, j\} \in e}=\left(A_{H}\right)_{i j}
$$

For the diagonal elements, we have $\left(X X^{\top}\right)_{i i}=\sum_{e \in E} X_{i e} X_{i e}=\operatorname{deg}(i)=d$. Therefore $A_{H}+d I=X X^{\top}$.

It's not hard to show that for $d \geqslant k$, all eigenvalues of $A_{G}$ from (13) occur in pairs $(\lambda,-\lambda)$, where $|\lambda|$ is a singular value of $X$, along with extra $(d n / k-n)$ many zero eigenvalues. The next result for random bipartite biregular graphs is given in [9].

Lemma 14 (Theorem 4 in [9]). Let $A_{G}$ be the adjacency matrix of a random bipartite biregular graph $G$ sampled uniformly from $\mathcal{G}\left(n, m, d_{1}, d_{2}\right)$, where $d_{1} \geqslant d_{2}$ are independent of $n$. Then:

1. Its second eigenvalue $\lambda_{2}$ satisfies

$$
\lambda_{2} \leqslant \sqrt{d_{1}-1}+\sqrt{d_{2}-1}+o(1)
$$

asymptotically almost surely as $n \rightarrow \infty$.

2. Its smallest positive eigenvalue $\lambda_{\min }^{+}$satisfies

$$
\lambda_{\min }^{+} \geqslant \sqrt{d_{1}-1}-\sqrt{d_{2}-1}-o(1) .
$$

We will use a result from [35] that estimates the probability that a random bipartite biregular graph sampled from $\mathcal{G}\left(n, m, d_{1}, d_{2}\right)$ contains some subgraph $L \subset K_{n, m}$, where $K_{n, m}$ is the complete bipartite graphs with $\left|V_{1}\right|=n,\left|V_{2}\right|=m$. Let $|L|$ be the number of edges of $L$ and we use the notation $[x]_{a}$ denotes the falling factorial $x(x-1) \cdots(x-a+1)$. For any vertex $v \in K_{n, m}$, let $g_{v}$ and $l_{v}$ denote the degree of $v$ considered as a vertex of $G$ and $L$ respectively. Let $l_{\max }$ be the largest value of $l_{i}$. 
Lemma 15 (Theorem 3.5 in [35]). Let $L \subset K_{n, m}$. If $|L|+2 d_{1}\left(d_{1}+l_{\max }-2\right) \leqslant n d_{1}-1$, then

$$
\mathbb{P}(L \subset G) \leqslant \frac{\prod\left[g_{i}\right]_{l_{i}}}{\left[n d_{1}-4 d_{1}^{2}-1\right]_{|L|}} .
$$

With Lemma 15, we are able to estimate the probability that a random bipartite biregular graph sampled uniformly from $\mathcal{G}(n, n d / k, d, k)$ belongs to $\mathcal{G}^{\prime}(n, n d / k, d, k)$.

Lemma 16. Let $G$ be a bipartite biregular graph sampled uniformly from $\mathcal{G}(n, n d / k, d, k)$ such that $3 \leqslant k \leqslant d \leqslant \frac{n}{32}$. Then

$$
\mathbb{P}\left(G \in \mathcal{G}^{\prime}(n, n d / k, d, k)\right)=1-O\left(\frac{d^{2}}{n k^{2}}\right) .
$$

In particular, if $3 \leqslant k \leqslant d \leqslant \frac{n}{32}$ and $\frac{d}{k}=o\left(n^{1 / 2}\right)$, as $n \rightarrow \infty$,

$$
\mathbb{P}\left(G \in \mathcal{G}^{\prime}(n, n d / k, d, k)\right) \rightarrow 1 \text {. }
$$

Proof. Let $V=V_{1} \cup V_{2}$ be the vertex set of a random graph $G$ sampled uniformly from $\mathcal{G}(n, n d / k, d, k)$. Assume there exist two vertices $v_{1}, v_{2} \in V_{2}$ such that $v_{1}, v_{2}$ have the same neighborhood in $V_{1}$ denoted by $N\left(v_{1}, v_{2}\right)$. Since $\operatorname{deg}\left(v_{1}\right)=\operatorname{deg}\left(v_{2}\right)=k, N\left(v_{1}, v_{2}\right)$ is of size $k$. Let $L$ be the subgraph induced by $N\left(v_{1}, v_{2}\right)$ and $v_{1}, v_{2}$ (see Figure 2). Then $|L|=2 k$ and $l_{\max }=k$. When $1 \leqslant d \leqslant \frac{n}{32}$, the assumption in Lemma 15 holds. By Lemma 15 , we have

$$
\mathbb{P}(L \subset G) \leqslant \frac{(d(d-1))^{k}\left([k]_{k}\right)^{2}}{\left[n d-4 d^{2}-1\right]_{2 k}} .
$$

The number of all possible vertex pairs in $V_{2}$ is $\left(\begin{array}{c}n d / k \\ 2\end{array}\right)$ and the number of all possible $k$ many distinct vertices in $V_{1}$ is $\left(\begin{array}{l}n \\ k\end{array}\right)$. Therefore for sufficiently large $n$, the probability that there exists two vertices in $V_{2}$ having the same neighborhood is at most

$$
\begin{aligned}
\left(\begin{array}{c}
n d / k \\
2
\end{array}\right) \cdot\left(\begin{array}{l}
n \\
k
\end{array}\right) \cdot \frac{d^{2 k} k^{2 k}}{\left[n d-4 d^{2}-1\right]_{2 k}} & \leqslant \frac{(n d)^{2}}{2 k^{2}}\left(\frac{n e}{k}\right)^{k}\left(\frac{d k}{n d-4 d^{2}-2 k}\right)^{2 k} \\
& \leqslant \frac{(n d)^{2}}{2 k^{2}}\left(\frac{n e}{k}\right)^{k}\left(\frac{2 k}{n}\right)^{2 k}=\frac{(n d)^{2}}{2 k^{2}}\left(\frac{4 e k}{n}\right)^{k} .
\end{aligned}
$$

Since $x \ln (x)$ is decreasing on $x \in\left(0, e^{-1}\right)$ and $3 \leqslant k \leqslant d \leqslant \frac{n}{32}$, we have for large $n$, $\frac{12 e}{n} \leqslant \frac{4 e k}{n}<e^{-1}$ and $k \ln (4 e k / n) \leqslant 3 \ln (12 e / n)$. Then $\left(\frac{4 e k}{n}\right)^{k} \leqslant\left(\frac{12 e}{n}\right)^{3}$. Therefore

$$
\mathbb{P}\left(G \notin \mathcal{G}^{\prime}(n, n d / k, d, k)\right)=O\left(\frac{d^{2}}{n k^{2}}\right) .
$$

This completes the proof. 
With the four lemmas above, we are ready to prove Theorem 11.

Proof of Theorem 11. Let $A_{H}$ be the adjacency matrix of a random $(d, k)$-regular hypergraph with $d \geqslant k$. Then its associated bipartite biregular graph has adjacency matrix (13), where $X$ is a $n \times n d / k$ matrix and $X X^{\top}=A_{H}+d I$. Let $G$ be a bipartite biregular graphs chosen uniformly from $\mathcal{G}(n, n d / k, d, k)$. From Lemma 16, we have

$$
\begin{aligned}
& \mathbb{P}\left(\lambda_{2}\left(A_{G}\right) \leqslant \sqrt{d-1}+\sqrt{k-1}+\epsilon_{n}\right) \\
= & \mathbb{P}\left(\lambda_{2}\left(A_{G}\right) \leqslant \sqrt{d-1}+\sqrt{k-1}+\epsilon_{n} \mid G \in \mathcal{G}^{\prime}(n, n d / k, d, k)\right) \\
& \cdot \mathbb{P}\left(G \in \mathcal{G}^{\prime}(n, n d / k, d, k)\right)+o(1) .
\end{aligned}
$$

By Lemma 14(1), asymptotically almost surely $\lambda_{2}\left(A_{G}\right) \leqslant \sqrt{d-1}+\sqrt{k-1}+\epsilon_{n}$ for some sequence $\epsilon_{n} \rightarrow 0$. Therefore by (16), we have

$$
\lim _{n \rightarrow \infty} \mathbb{P}\left(\lambda_{2}\left(A_{G}\right) \leqslant \sqrt{d-1}+\sqrt{k-1}+\epsilon_{n} \mid G \in \mathcal{G}^{\prime}(n, n d / k, d, k)\right)=1 .
$$

The uniform measure on $\mathcal{G}(n, n d / k, d, k)$ conditioned on the event $\left\{G \in \mathcal{G}^{\prime}(n, n d / k, d, k)\right\}$ is a uniform measure on $\mathcal{G}^{\prime}(n, n d / k, d, k)$. Hence asymptotically almost surely a bipartite biregular graph $G$ sampled uniformly from $\mathcal{G}^{\prime}(n, n d / k, d, k)$ satisfies (14).

Note that $G$ also satisfies (15) asymptotically almost surely. Since there is a bijection between $\mathcal{G}^{\prime}(n, n d / k, d, k)$ and $\mathcal{H}(n, d, k)$ described in Lemma 10, by (14) and Lemma 13, we have with high probability, $\lambda_{2}\left(X X^{\top}\right)=\lambda_{2}^{2}\left(A_{G}\right) \leqslant d+k-2+2 \sqrt{(k-1)(d-1)}+o(1)$. And it implies with high probability,

$$
\lambda_{2}\left(A_{H}\right)-k+2 \leqslant 2 \sqrt{(k-1)(d-1)}+o(1) .
$$

Similarly, from (15), for the smallest eigenvalue $\lambda_{n}\left(A_{H}\right)$, we have with high probability,

$$
\lambda_{n}\left(A_{H}\right)+d=\lambda_{n}\left(X X^{\top}\right)=\lambda_{\min }^{+}\left(A_{G}\right)^{2} \geqslant d+k-2-2 \sqrt{(d-1)(k-1)}-o(1),
$$

which implies with high probability,

$$
\lambda_{n}\left(A_{H}\right)-k+2 \geqslant-2 \sqrt{(d-1)(k-1)}-o(1) .
$$

Combining (17) with (18), and note that the largest eigenvalue of $A$ is $d(k-1)$, we have $|\lambda-k+2| \leqslant 2 \sqrt{(d-1)(k-1)}+o(1)$ for any eigenvalue $\lambda \neq d(k-1)$ asymptotically almost surely. This completes the proof of Theorem 11 .

\section{Spectra of the non-backtracking operators}

Following the definition in [3], for a hypergraph $H=(V, E)$, its non-backtracking operator $B$ is a square matrix indexed by oriented hyperedges $\vec{E}=\{(i, e): i \in V, e \in E, i \in e\}$ with entries given by

$$
B_{(i, e),(j, f)}= \begin{cases}1 & \text { if } j \in e \backslash\{i\}, f \neq e \\ 0 & \text { otherwise }\end{cases}
$$


for any oriented hyperedges $(i, e),(j, f)$. This is a generalization of the graph nonbacktracking operators to hypergraphs. In [3], a spectral algorithm was proposed for solving community detection problems on sparse random hypergraph, and it uses the eigenvectors of the non-backtracking operator defined above. To obtain theoretical guarantees for this spectral algorithm, we need to prove a spectral gap for the non-backtracking operator. To the best of our knowledge, this operator has not been rigorously analyzed for any random hypergraph models. In the first step, we study the spectrum of the non-backtracking operator for the random regular hypergraphs. From the bijection in Lemma 10, it is important to find its connection to the non-backtracking operator of the corresponding bipartite biregular graph.

Consider a bipartite graph $G=(V(G), E(G))$ with $V(G)=V_{1}(G) \cup V_{2}(G)$. The non-backtracking operator $B_{G}$ of $G$ is a matrix indexed by the set of oriented edges $\vec{E}(G)=\{e=(i, j): i, j \in V(G), e \in E(G)\}$ with dimension $2|E(G)| \times 2|E(G)|$. For an oriented edge $e=(i, j)$ and $f=(s, t)$, define $B_{G}$ as

$$
\left(B_{G}\right)_{e f}= \begin{cases}1, & \text { if } j=s \text { and } t \neq i \\ 0, & \text { otherwise }\end{cases}
$$

We order the elements of $\vec{E}$ as $\left\{e_{1}, \ldots, e_{2|E(G)|}\right\}$, so that the first $|E(G)|$ oriented edges have starting vertices from $V_{1}$ and ending vertices in $V_{2}$. In this way, we can write

$$
B_{G}=\left(\begin{array}{cc}
0 & M \\
N & 0
\end{array}\right)
$$

where $M, N$ are $|E| \times|E|$ matrices with entries in $\{0,1\}$. The following lemma connects the non-backtracking operator $B_{H}$ of a hypergraph $H$ to the non-backtracking operator $B_{G}$ of its associated bipartite graph $G$.

Lemma 17. Let $B_{H}$ be a non-backtracking operator of $H$. Let $G$ be its associated bipartite graph with a non-backtracking operator given by (19). Then $B_{H}=M N$.

Proof. Since $B_{G}^{2}=\left(\begin{array}{cc}M N & 0 \\ 0 & N M\end{array}\right)$, it suffices to show the $|E| \times|E|$ submatrix $M N$ in $B_{G}^{2}$ is $B_{H}$. From our construction of the associated bipartite graph, we know $V(G)=V_{1} \cup V_{2}$ and $V_{1}=V(H), V_{2}=E(H)$. The oriented edges with starting vertices from $V_{1}$ and ending vertices from $V_{2}$ can be denoted by $(i, e)$, where $i \in V(H), e \in E(H)$. Then for any $(i, e),(j, f)$ in $\vec{E}(G)$, we have

$$
\begin{aligned}
\left(B_{G}^{2}\right)_{(i, e),(j, f)} & =\sum_{(k, g) \in \vec{E}(G)}\left(B_{G}\right)_{(i, e),(k, g)}\left(B_{G}\right)_{(k, g),(j, f)}=\sum_{(k, g) \in \vec{E}(G)} \mathbf{1}_{\{e=k, g \neq i\}} \mathbf{1}_{\{j=g, f \neq k\}} \\
& =\mathbf{1}_{\{(e, j) \in \vec{E}(G)\}} \mathbf{1}_{\{j \neq i, f \neq e\}}=\mathbf{1}_{\{j \in e, j \neq i, f \neq e\}}=\left(B_{H}\right)_{(i, e),(j, f)} .
\end{aligned}
$$

Hence $B_{H}=M N$, this completes the proof.

Remark 18. Lemma 17 is true for any hypergraphs, including non-uniform hypergraphs. 
If $H$ is a $(d, k)$-regular hypergraph, then $G$ is a $(d, k)$-bipartite biregular graph with $\left|V_{1}(G)\right|=n,\left|V_{2}(G)\right|=n d / k$. Our next lemma for the spectrum of $B_{G}$ is from from [9].

Lemma 19 (Lemma 2 in [9]). Let $G$ be a $(d, k)$-bipartite biregular graph with $n$ vertices. Any eigenvalue of $B_{G}$ belongs to one of the following categories:

1. \pm 1 are both eigenvalues with multiplicity $|E(G)|-|V(G)|=n(d-1)-n d / k$.

2. $\pm i \sqrt{d-1}$ are eigenvalues with multiplicity $n-r$, where $r$ is the rank of $X$.

3. $\pm i \sqrt{k-1}$ are eigenvalues with multiplicity $n d / k-r$.

4. Every pair of non-zero eigenvalues $(-\xi, \xi)$ of the adjacency matrix $A_{G}$ generates exactly 4 eigenvalues of $B_{G}$ with the equation $\lambda^{4}-\left(\xi^{2}-d-k+2\right) \lambda^{2}+(k-1)(d-1)=0$.

We have the following characterization of eigenvalues for $B_{H}$ of a $(d, k)$-regular hypergraph $H$. It follows immediately from Lemma 17 and Lemma 19.

Theorem 20. Let $H$ be $a(d, k)$-regular hypergraph on $n$ vertices and $G$ be its associated $(d, k)$-bipartite biregular graph with adjacency matrix $A_{G}$ given in (13). All eigenvalues of $B_{H}$ can be classified into the following:

1. 1 with multiplicity $n(d-1)-n d / k$.

2. $-(d-1)$ with multiplicity $n-r$, where $r$ is the rank of $X$.

3. $-(k-1)$ with multiplicity $n d / k-r$.

4. Every pair of non-zero eigenvalues $(-\xi, \xi)$ of $A_{G}$ generates exactly 2 eigenvalues of $B_{H}$ with the equation: $\lambda^{2}-\left(\xi^{2}-d-k-2\right) \lambda+(k-1)(d-1)=0$.

Let $G$ be an associated $(d, k)$-bipartite biregular graph of a regular hypergraph $H$. From [9, Section 2], $\pm \sqrt{(d-1)(k-1)}$ are eigenvalues of $B_{G}$ with multiplicity 1 . Then from Theorem 20, $B_{H}$ has an eigenvalue $\lambda_{1}\left(B_{H}\right)=(d-1)(k-1)$ with multiplicity 1 . From $[9$, Theorem 3], for random $(d, k)$-bipartite biregular graphs, the second largest eigenvalue (in absolute value) $\lambda_{2}\left(B_{G}\right)$ satisfies

$$
\left|\lambda_{2}\left(B_{G}\right)\right| \leqslant((k-1)(d-1))^{1 / 4}+o(1)
$$

asymptotically almost surely as $n \rightarrow \infty$. Therefore from the discussion above, together with Lemma 16, we obtain the following spectral gap result for $B_{H}$.

Theorem 21. Let $H$ be a random $(d, k)$-regular hypergraph sampled from $\mathcal{H}(n, d, k)$, with $d \geqslant k \geqslant 3$. Then any eigenvalue $\lambda$ of $B_{H}$ with $\lambda \neq(d-1)(k-1)$ satisfies

$$
|\lambda| \leqslant((k-1)(d-1))^{1 / 2}+o(1)
$$

asymptotically almost surely as $n \rightarrow \infty$. 


\section{Empirical spectral distributions}

In the last section, we study the empirical spectral distribution of the adjacency matrix of a random regular hypergraph. We define the empirical spectral distribution (ESD) of a symmetric $n \times n$ matrix $M$ to be the probability measure $\mu_{n}$ on $\mathbb{R}$ given by

$$
\mu_{n}=\frac{1}{n} \sum_{i=1} \delta_{\lambda_{i}}
$$

where $\delta_{x}$ is the point mass at $x$ and $\lambda_{1}, \ldots, \lambda_{n}$ are the eigenvalues of $M$. We always assume $d \geqslant k$ (see Remark 9). Feng and Li in [16] derived the limiting ESD for a sequence of connected $(d, k)$-regular hypergraphs with fixed $d, k$ as follows. The definition of primitive cycles in [16] is the same as cycles in our Definition 2.

Theorem 22 (Theorem 4 in [16]). Let $H_{n}$ be a family of connected $(d, k)$-regular hypergraphs with $n$ vertices. Assume for each integer $l \geqslant 1$, the number of cycles of length $l$ is $o(n)$. Denote $q=(d-1)(k-1)$. For fixed $d \geqslant k \geqslant 3$, the empirical spectral distribution of $M_{n}:=\frac{A-(k-2)}{\sqrt{(d-1)(k-1)}}$ converges weakly in probability to the measure $\mu$ supported on $[-2,2]$, whose density function is given by

$$
f(x):=\frac{1+\frac{k-1}{q}}{\left(1+\frac{1}{q}-\frac{x}{\sqrt{q}}\right)\left(1+\frac{(k-1)^{2}}{q}+\frac{(k-1) x}{\sqrt{q}}\right)} \cdot \frac{1}{\pi} \sqrt{1-\frac{x^{2}}{4}} d x .
$$

We prove that for uniform random regular hypergraphs, the assumptions in Theorem 22 hold with high probability, which implies the convergence of ESD in probability for random regular hypergraphs.

Lemma 23. Let $H$ be a random $(d, k)$-regular hypergraph with fixed $d \geqslant k \geqslant 3$. Then $H$ is connected asymptotically almost surely.

Proof. $H$ is connected if and only if its associated bipartite biregular graph $G$ is connected. The first eigenvalue for the $(d, k)$-bipartite biregular graph $G$ is $\lambda_{1}=\sqrt{d k}$ and we know from Lemma 14 and Lemma 16, for a uniformly chosen random regular hypergraph $H$, the corresponding bipartite biregular graph $G$ satisfies $\lambda_{2} \leqslant \sqrt{d-1}+\sqrt{k-1}+o(1)$ asymptotically almost surely. Note that for $d, k \geqslant 2, \sqrt{d-1}+\sqrt{k-1}=\sqrt{d k}$ if and only if $d=k=2$. So when $d \geqslant k \geqslant 3$, for sufficiently large $n$, the first eigenvalue has multiplicity one with high probability. If $G$ is not connected, we can decompose $G$ as $G=G_{1} \cup G_{2}$ such that there is no edge between $G_{1}$ and $G_{2}$. Then $G_{1}, G_{2}$ are both bipartite biregular graphs with the largest eigenvalue $\sqrt{d k}$. However, that implies $G$ satisfies $\lambda_{2}=\sqrt{d k}$, a contradiction.

The following lemma shows the number of cycles of length $l$ in $H$ is $o(n)$ asymptotically almost surely.

Lemma 24. Let $H_{n}$ be a random $(d, k)$-regular hypergraph. For each integer $l \geqslant 1$, the number of cycles of length $l$ in $H_{n}$ is o(n) asymptotically almost surely. 
Proof. By Lemma 16, it is equivalent to show the number of cycles of length $2 l$ for a random bipartite biregular graph, denoted by $X_{l}$, is $o(n)$ with high probability. From [15, Proposition 4], when $d, k, l$ are fixed, $\mathbb{E}\left[X_{l}\right]=O(1)$, and $\operatorname{Var}\left[X_{l}\right]=O(1)$. Then by Chebyshev's inequality,

$$
\mathbb{P}\left(\left|X_{l}-\mathbb{E} X_{l}\right| \geqslant \frac{n}{\log n}\right)=O\left(\frac{\log ^{2}(n)}{n^{2}}\right) .
$$

Hence $X_{l}=o(n)$ asymptotically almost surely.

Combining Theorem 22, Lemma 23 and Lemma 24, we have the following theorem for the ESDs of random regular hypergraphs with fixed $d, k$ :

Theorem 25. Let $A_{n}$ be the adjacency matrix of a random $(d, k)$-regular hypergraph on $n$ vertices. Let $M_{n}:=\frac{A-(k-2)}{\sqrt{(d-1)(k-1)}}$. For fixed $d \geqslant k \geqslant 3$, the empirical spectral distribution of $M_{n}$ converges in probability to a measure $\mu$ with density function $f(x)$ given in $(21)$.

Remark 26. When $k=2, f(x)$ is the density of the Kesten-McKay law [34] with a different scaling factor. For $k \geqslant 3$, the limiting distribution in (21) is not symmetric (i.e. $f(x) \neq f(-x)$ ), which is quite different from the random graph case. For random bipartite biregular graphs with bounded degrees, the limit of the ESDs was derived in [19], and later in [7] using different methods.

In [16], the cases where $d, k$ grow with $n$ have not been discussed. With the results on random bipartite biregular graphs from $[15,41]$, we can get the following result in this regime.

Theorem 27. Let $A_{n}$ be the adjacency matrix of a random $(d, k)$-regular hypergraph on $n$ vertices. For $d \rightarrow \infty$ with $\frac{d}{k} \rightarrow \alpha \geqslant 1$ and $d=o\left(n^{1 / 2}\right)$, the empirical spectral distribution of $M_{n}:=\frac{A_{n}-(k-2)}{\sqrt{(d-1)(k-1)}}$ converges in probability to a measure supported on $[-2,2]$ with a density function

$$
g(x)=\frac{\alpha}{1+\alpha+\sqrt{\alpha} x} \frac{1}{\pi} \sqrt{1-\frac{x^{2}}{4}} .
$$

To prove Theorem 27, we will apply the following results for the global law of random bipartite biregular graphs.

Theorem 28 (Theorem 1 in [15] and Corollary 2.2 in [41]). Let $A_{G}$ be the adjacency matrix of a random bipartite biregular graph sampled from $\mathcal{G}(n, m, d, k)$ with $n \leqslant m$, $\frac{d}{k} \rightarrow \alpha \geqslant 1$, and $d=o\left(n^{1 / 2}\right)$ as $n \rightarrow \infty$. Then the ESD of $\frac{A_{G}}{\sqrt{k}}$ converges asymptotically almost surely to a distribution supported on $[-b,-a] \cup[a, b]$ with density

$$
h(x):=\frac{\alpha}{(1+\alpha) \pi|x|} \sqrt{\left(b^{2}-x^{2}\right)\left(x^{2}-a^{2}\right)},
$$

and a point mass of $\frac{\alpha-1}{\alpha+1}$ at 0 , where $a=1-\alpha^{-1 / 2}, b=1+\alpha^{-1 / 2}$. 
Proof of Theorem 27. Let $A_{G}$ be the adjacency matrix of a random $(d, k)$-bipartite biregular graph sampled from $\mathcal{G}(n, n d / k, d, k)$. Since $X$ is a $n \times m$ matrix with $n \leqslant m$, the ESD of $\frac{X X^{\top}}{k}$ is the distribution of the squares of the nonzero eigenvalues of $\frac{A_{G}}{\sqrt{k}}$, and from (23), the ESD of $\frac{X X^{\top}}{k}$ is supported on $\left[a^{2}, b^{2}\right]$ with the density function given by

$$
\tilde{h}(x)=\frac{\alpha}{2 \pi x} \sqrt{\left(b^{2}-x\right)\left(x-a^{2}\right)}
$$

asymptotically almost surely. By Lemma 16, the same statement holds for a random bipartite biregular graph $G$ sampled uniformly from $\mathcal{G}^{\prime}(n, n d / k, d, k)$. Since the adjacency matrix of the corresponding regular hypergraph $H$ is $A_{n}=X X^{\top}-d$, by scaling, this implies that the ESD of $M_{n}=\frac{A_{n}-k-2}{\sqrt{(d-1)(k-1)}}$ is supported on $[-2,2]$ and the density is given by $(22)$.

The convergence of empirical spectral distributions on short intervals (also known as the local law) for random bipartite biregular graphs was studied in $[15,41,44]$. Universality of eigenvalue statistics was studied in [43]. All of these local eigenvalue statistics can be translated to random regular hypergraphs via the bijection in Lemma 10. As an example, we translate the following result about the local law for random bipartite biregular graphs in [15] to random regular hypergraphs.

Theorem 29. Let $H$ be a random $(d, k)$-regular hypergraph on $n$ vertices satisfying $d \rightarrow \infty$ as $n \rightarrow \infty, \frac{d}{k} \rightarrow \alpha \geqslant 1$ and $\log k=o(\sqrt{\log n})$. Let $A$ be the adjacency matrix of $H$ and $\mu_{n}$ be the ESD of $M:=\frac{A-d}{\sqrt{(d-1)(k-1)}}$ and $\mu$ be the limiting ESD defined in (22). For any $\epsilon>0$, there exists a constant $C_{\epsilon}$ such that for all sufficiently large $n$ and $\delta>0$, for any interval $I \subset[-2+\epsilon, 2]$ with length $|I| \geqslant \frac{4(1+\sqrt{\alpha})^{2}}{\sqrt{\alpha}} \max \left\{2 \eta, \frac{\eta}{-\delta \log \delta}\right\}$, it holds that $\left|\mu_{n}(I)-\mu(I)\right| \leqslant \delta C_{\epsilon}|I|$ with probability $1-o(1)$, where $\eta$ is given by the following quantities:

$$
h=\min \left\{\frac{\log n}{9(\log k)^{2}}, k\right\}, \quad r=e^{1 / h}, \quad \eta=r^{1 / 2}-r^{-1 / 2} .
$$

We prove Theorem 29 from the following local law for random bipartite biregular graphs in [15].

Lemma 30 (Theorem 3 in [15]). Let $G$ be a random $(d, k)$-bipartite biregular graph on $n+n d / k$ vertices satisfying $d \rightarrow \infty$ as $n \rightarrow \infty$ and $\log k=o(\sqrt{\log n}), \frac{d}{k} \rightarrow \alpha \geqslant 1$. Let $A_{G}$ be the adjacency matrix of $G$ and $\mu_{n}$ be the ESD of $\frac{A_{G}}{\sqrt{k-1}}$ and let $\mu$ be the measure defined in (23). For any $\epsilon>0$, there exists a constant $C_{\epsilon}$ such that for all sufficiently large $n$ and $0<\delta<1$, for any interval $I \subset \mathbb{R}$ avoiding $[-\epsilon, \epsilon]$ and with length $|I| \geqslant \max \left\{2 \eta, \frac{\eta}{-\delta \log \delta}\right\}$,

$$
\left|\mu_{n}(I)-\mu(I)\right| \leqslant \delta C_{\epsilon}|I|
$$

with probability $1-o(1 / n)$, where $\eta$ is given in (25). 
Proof of Theorem 29. For any interval $I \subset \mathbb{R}$ and a symmetric matrix $M$, we denote $N_{I}^{M}$ to be the number of eigenvalues of $M$ in the interval $I$. For a random $(d, k)$-regular hypergraph $H$ with adjacency matrix $A$, let $G$ be its associated bipartite biregular graph and $A_{G}$ be the adjacency matrix of $G$. With Lemma 16, we know (26) holds for $A_{G}$ with probability $1-o(1)$. Recall that the ESD of $W:=\frac{X X^{\top}}{k-1}$ is the distribution of the squares of the nontrivial eigenvalues of $\frac{A_{G}}{\sqrt{k-1}}$. Let $M:=\frac{A_{G}-k-2}{\sqrt{(d-1)(k-1)}}$. Consider any interval $I_{1}=[\beta, \gamma] \subset[-2+\epsilon, 2]$ with length

$$
\left|I_{1}\right| \geqslant \frac{4(1+\sqrt{\alpha})^{2}}{\sqrt{\alpha}} \max \left\{2 \eta, \frac{\eta}{-\delta \log \delta}\right\} .
$$

Let $I_{2}=\left[\sqrt{\frac{d-1}{k-1}} \beta+\frac{d+k+2}{k-1}, \sqrt{\frac{d-1}{k-1}} \gamma+\frac{d+k+2}{k-1}\right]:=\left[\beta^{\prime}, \gamma^{\prime}\right]$ be a shifted and rescaled interval from $I_{1}$. We have

$$
\begin{aligned}
\left|I_{2}\right| & =\gamma^{\prime}-\beta^{\prime}=(\sqrt{\alpha}+o(1))(\beta-\gamma)=(\sqrt{\alpha}+o(1))\left|I_{1}\right|, \\
\beta^{\prime} & =\sqrt{\alpha} \beta+\alpha+1+o(1)=(\sqrt{\alpha}-1)^{2}+\sqrt{\alpha} \epsilon+o(1)>(\sqrt{\alpha}-1)^{2}+\frac{\epsilon}{2}, \\
\gamma^{\prime} & \leqslant 2 \sqrt{\frac{d-1}{k-1}}+\frac{d+k+2}{k-1}=2 \sqrt{\alpha}+\alpha+1+o(1)=(1+\sqrt{\alpha})^{2}+o(1) .
\end{aligned}
$$

Let $I_{3}:=\left[\sqrt{\beta^{\prime}}, \sqrt{\gamma^{\prime}}\right.$. From the eigenvalue relation between $M, W$ and $A_{G}$, we have $N_{I_{1}}^{M}=N_{I_{2}}^{W}=2 N_{I_{3}}^{\frac{A_{G}}{\sqrt{k-1}}}$. Note that from (28) and (30), the interval length of $I_{3}$ satisfies

$$
\left|I_{3}\right|=\sqrt{\gamma^{\prime}}-\sqrt{\beta^{\prime}}=\frac{\gamma^{\prime}-\beta^{\prime}}{\sqrt{\gamma^{\prime}}+\sqrt{\beta^{\prime}}} \geqslant \frac{(\sqrt{\alpha}+o(1))\left|I_{1}\right|}{2(1+\sqrt{\alpha})^{2}+o(1)} \geqslant \max \left\{2 \eta, \frac{\eta}{-\delta \log \delta}\right\}
$$

where the last inequality is from (27). From (29),

$$
\left|I_{3}\right|=\frac{\gamma^{\prime}-\beta^{\prime}}{\sqrt{\gamma^{\prime}}+\sqrt{\beta^{\prime}}} \leqslant \frac{\gamma^{\prime}-\beta^{\prime}}{2 \sqrt{\beta^{\prime}}} \leqslant \frac{\sqrt{\alpha}+o(1)}{2(\sqrt{\alpha}-1)^{2}+\epsilon}\left|I_{1}\right| .
$$

From Lemma 30 , since $\sqrt{\beta^{\prime}} \geqslant \sqrt{\epsilon / 2}, I_{3}$ is an interval avoiding $[-\sqrt{\epsilon / 2}, \sqrt{\epsilon / 2}]$, hence there exists a constant $C_{\epsilon}$ such that

$$
\left|\frac{1}{n+\frac{n d}{k}} N_{I_{3}}^{\frac{A_{G}}{\sqrt{k-1}}}-\mu_{G}\left(I_{3}\right)\right| \leqslant \delta C_{\epsilon}\left|I_{3}\right|,
$$

where $\mu_{G}$ is the limiting measure defined in (23). Let $\mu_{X}$ be the limiting measure defined in (24) and $\mu_{A}$ be the limiting measure defined in (22). Note that $\mu_{A}\left(I_{1}\right)=\mu_{X}\left(I_{2}\right)=$ $2(\alpha+1) \mu_{G}\left(I_{3}\right)$. Therefore (32) implies

$$
\left|\frac{1}{2\left(n+\frac{n d}{k}\right)} N_{I_{1}}^{M}-\frac{1}{2(\alpha+1)} \mu_{A}\left(I_{1}\right)\right| \leqslant \delta C_{\epsilon}\left|I_{3}\right| .
$$


Let $\mu_{n}$ be the ESD of $M$. From (33), we get

$$
\begin{aligned}
& \left|\mu_{n}\left(I_{1}\right)-\mu_{A}\left(I_{1}\right)\right|=\left|\frac{1}{n} N_{I_{1}}^{M}-\mu_{A}\left(I_{1}\right)\right|=2\left(1+\frac{d}{k}\right)\left|\frac{1}{2\left(n+\frac{n d}{k}\right)} N_{I_{1}}^{M}-\frac{1}{2\left(1+\frac{d}{k}\right)} \mu_{A}\left(I_{1}\right)\right| \\
\leqslant & 2\left(1+\frac{d}{k}\right)\left(\left|\frac{1}{2\left(n+\frac{n d}{k}\right)} N_{I_{1}}^{M}-\frac{1}{2(1+\alpha)} \mu_{A}\left(I_{1}\right)\right|+\left|\frac{1}{2(\alpha+1)}-\frac{1}{2\left(1+\frac{d}{k}\right)}\right| \mu_{A}\left(I_{1}\right)\right) \\
\leqslant & 2\left(1+\frac{d}{k}\right) \delta C_{\epsilon}\left|I_{3}\right|+\frac{1}{\alpha+1}\left|\frac{d}{k}-\alpha\right| \mu_{A}\left(I_{1}\right) \\
\leqslant & \delta \frac{(2+2 \alpha) \sqrt{\alpha}+o(1)}{2(\sqrt{\alpha}-1)^{2}+\epsilon} C_{\epsilon}\left|I_{1}\right|+o\left(\mu_{A}\left(I_{1}\right)\right) \leqslant \frac{4 \delta(1+\alpha) \sqrt{\alpha}}{2(\sqrt{\alpha}-1)^{2}+\epsilon} C_{\epsilon}\left|I_{1}\right|:=\delta C_{\epsilon}^{\prime}\left|I_{1}\right|
\end{aligned}
$$

where the first inequality in (34) is from (31), and $C_{\epsilon}^{\prime}$ is a constant depending on $\alpha$ and

$\epsilon$. This completes the proof of Theorem 29.

\section{Acknowledgments}

We thank Sebastian Cioabă and Kameron Decker Harris for helpful comments. This work was partially supported by NSF DMS-1949617.

\section{References}

[1] Noga Alon. Eigenvalues and expanders. Combinatorica, 6(2):83-96, 1986.

[2] Noga Alon, Itai Benjamini, Eyal Lubetzky, and Sasha Sodin. Non-backtracking random walks mix faster. Communications in Contemporary Mathematics, 9(04):585603, 2007.

[3] Maria Chiara Angelini, Francesco Caltagirone, Florent Krzakala, and Lenka Zdeborová. Spectral detection on sparse hypergraphs. In Communication, Control, and Computing (Allerton), 2015 53rd Annual Allerton Conference on, pages 66-73. IEEE, 2015.

[4] Anirban Banerjee. On the spectrum of hypergraphs. Linear Algebra and its Applications, 614:82-110, 2021.

[5] Claude Berge. Graphs and Hypergraphs. North-Holland mathematical library. Amsterdam, 1973.

[6] Charles Bordenave. A new proof of Friedman's second eigenvalue theorem and its extension to random lifts. arXiv:1502.04482, 2015.

[7] Charles Bordenave and Marc Lelarge. Resolvent of large random graphs. Random Structures \& Algorithms, 37(3):332-352, 2010.

[8] Gerandy Brito, Ioana Dumitriu, Shirshendu Ganguly, Christopher Hoffman, and Linh V Tran. Recovery and rigidity in a regular stochastic block model. In Proceedings 
of the twenty-seventh annual ACM-SIAM symposium on Discrete algorithms, pages 1589-1601. Society for Industrial and Applied Mathematics, 2016.

[9] Gerandy Brito, Ioana Dumitriu, and Kameron Decker Harris. Spectral gap in random bipartite biregular graphs and its applications. arXiv:1804.07808, 2018.

[10] Fan Chung. Spectral graph theory. Number 92 in CBMS Regional Conference Series. American Mathematical Society, 1997.

[11] Sebastian M Cioabă and Peng Xu. Mixing rates of random walks with little backtracking. Contemporary Mathematics, 655, 2015.

[12] Emma Cohen, Dhruv Mubayi, Peter Ralli, and Prasad Tetali. Inverse expander mixing for hypergraphs. The Electronic Journal of Combinatorics, 23(2):\#P2.20, 2016.

[13] Colin Cooper, Alan Frieze, and Tomasz Radzik. The cover times of random walks on random uniform hypergraphs. Theoretical Computer Science, 509:51-69, 2013.

[14] Simon Coste. The spectral gap of sparse random digraphs. Annales de l'Institut Henri Poincaré, Probabilités et Statistiques, 57(2):644-684, 2021.

[15] Ioana Dumitriu and Tobias Johnson. The Marčenko-Pastur law for sparse random bipartite biregular graphs. Random Structures \& Algorithms, 48(2):313-340, 2016.

[16] Keqin Feng and Wen-Ching Winnie Li. Spectra of hypergraphs and applications. Journal of number theory, 60(1):1-22, 1996.

[17] Joel Friedman. A proof of Alon's second eigenvalue conjecture. In Proceedings of the thirty-fifth annual ACM symposium on Theory of computing, pages 720-724. ACM, 2003.

[18] Joel Friedman and Avi Wigderson. On the second eigenvalue of hypergraphs. Combinatorica, 15(1):43-65, 1995.

[19] Chris D Godsil and Bojan Mohar. Walk generating functions and spectral measures of infinite graphs. Linear Algebra and its Applications, 107:191-206, 1988.

[20] Amine Helali and Matthias Löwe. Hitting times, commute times, and cover times for random walks on random hypergraphs. Statistics $\& 5$ Probability Letters, 154:108535, 2019.

[21] Christopher J Hillar and Lek-Heng Lim. Most tensor problems are NP-hard. Journal of the ACM (JACM), 60(6):45, 2013.

[22] John Lenz and Dhruv Mubayi. Eigenvalues and linear quasirandom hypergraphs. In Forum of Mathematics, Sigma, volume 3. Cambridge University Press, 2015.

[23] John Lenz and Dhruv Mubayi. Eigenvalues of non-regular linear quasirandom hypergraphs. Discrete Mathematics, 340(2):145-153, 2017.

[24] Hong-Hai Li and Bojan Mohar. On the first and second eigenvalue of finite and infinite uniform hypergraphs. Proceedings of the American Mathematical Society, 147(3):933-946, 2019. 
[25] Wen-Ching Winnie Li. Ramanujan hypergraphs. Geometric and Functional Analysis, 14(2):380-399, 2004.

[26] Wen-Ching Winnie Li and Patrick Solé. Spectra of regular graphs and hypergraphs and orthogonal polynomials. European Journal of Combinatorics, 17(5):461-477, 1996.

[27] Qingshan Liu, Yuchi Huang, and Dimitris N Metaxas. Hypergraph with sampling for image retrieval. Pattern Recognition, 44(10-11):2255-2262, 2011.

[28] Ying Liu, Jiabin Yuan, Bojia Duan, and Dan Li. Quantum walks on regular uniform hypergraphs. Scientific reports, 8(1):9548, 2018.

[29] László Lovász. Random walks on graphs: a survey. Combinatorics, Paul Erdős is Eighty, 2:353-398, 1994.

[30] Linyuan Lu and Xing Peng. High-ordered random walks and generalized Laplacians on hypergraphs. In International Workshop on Algorithms and Models for the WebGraph, pages 14-25. Springer, 2011.

[31] Linyuan Lu and Xing Peng. Loose Laplacian spectra of random hypergraphs. Random Structures \& Algorithms, 41(4):521-545, 2012.

[32] Alexander Lubotzky, Ralph Phillips, and Peter Sarnak. Ramanujan graphs. Combinatorica, 8(3):261-277, 1988.

[33] María G Martínez, Harold M Stark, and Audrey Terras. Some Ramanujan hypergraphs associated to $G L\left(n, F_{q}\right)$. Proceedings of the American Mathematical Society, 129(6):1623-1629, 2001.

[34] Brendan D McKay. The expected eigenvalue distribution of a large regular graph. Linear Algebra and its Applications, 40:203-216, 1981.

[35] Brendan D McKay. Subgraphs of random graphs with specified degrees. In Congressus Numerantium, volume 33, pages 213-223, 1981.

[36] Marina Meilă and Jianbo Shi. A random walks view of spectral segmentation. In International Workshop on Artificial Intelligence and Statistics, pages 203-208. PMLR, 2001.

[37] Alireza Sarveniazi. Explicit construction of a Ramanujan $\left(n_{1}, n_{2}, \ldots, n_{d-1}\right)$-regular hypergraph. Duke Mathematical Journal, 139(1):141-171, 2007.

[38] Christopher K Storm. The Zeta function of a hypergraph. the Electronic Journal of Combinatorics, 13(1):84, 2006.

[39] Shulong Tan, Jiajun Bu, Chun Chen, Bin Xu, Can Wang, and Xiaofei He. Using rich social media information for music recommendation via hypergraph model. ACM Transactions on Multimedia Computing, Communications, and Applications (TOMM), 7(1):22, 2011.

[40] Ze Tian, Tae Hyun Hwang, and Rui Kuang. A hypergraph-based learning algorithm for classifying gene expression and arraycgh data with prior knowledge. Bioinformatics, 25(21):2831-2838, 2009. 
[41] Linh V Tran. Local law for eigenvalues of random regular bipartite graphs. Bulletin of the Malaysian Mathematical Sciences Society, 43(2):1517-1526, 2020.

[42] Yali Wan and Marina Meila. A class of network models recoverable by spectral clustering. In Advances in Neural Information Processing Systems, pages 3285-3293, 2015.

[43] Kevin Yang. Bulk eigenvalue correlation statistics of random biregular bipartite graphs. arXiv:1705.00083, 2017.

[44] Kevin Yang. Local Marchenko-Pastur law for random bipartite graphs. arXiv preprint arXiv:1704.08672, 2017.

[45] Denny Zhou, Jiayuan Huang, and Bernhard Schölkopf. Learning with hypergraphs: Clustering, classification, and embedding. In Advances in neural information processing systems, pages 1601-1608, 2007. 